through the clinical audit department and patients' case notes were examined to review attributes of error.

Results There were 38 incidents over the period of review (3.2 errors per month), demonstrating an improvement from the last audit cycle (4.5 errors per month). A large percentage $(68 \%)$ of errors originated from paediatric ward and most of the errors were attributable to prescription errors. Doctors of all grades were identified to commit errors in prescription. Administration issues were the second most common cause for errors. Neither of these errors resulted in mortality and morbidity although some patients required additional monitoring as a consequence.

Conclusions It is important to note that while errors were reducing in frequency, these errors could still potentially cause harm if they were not being addressed. Our trust is currently in the process of rolling out electronic prescribing and administration of medications to reduce medication errors and improve patient care. Another audit will be planned after the implementation of electronic system to review its usage to improve medication safety.

\section{MAXIMISING THE EDUCATIONAL VALUE OF CLERKING FOR JUNIOR TRAINEES ON A PAEDIATRIC CARDIOLOGY WARD}

Emily Operto, Esme Dunne. UK

\subsection{6/bmjpo-2021-RCPCH.31}

Background The cardiorespiratory physiology of congenital heart defects can be complex and junior trainees infrequently receive training in echocardiogram interpretation. A rotation in paediatric cardiology provides a wealth of learning opportunities however these may not be realised because of service pressures.

Objectives This quality improvement project aimed to maximise the educational opportunities of clerking ward admissions for junior trainees on a paediatric cardiology ward. The two structured interventions focussed on tandem interpretation of patients' cardiorespiratory physiology and echocardiogram findings, between junior and senior trainees, with the aim of encouraging learning and feedback.

Methods An anonymous questionnaire of six junior trainees collected baseline quantitative data to assess educational aspects of clerking admissions on a cardiology ward in a paediatric cardiology centre in London. Two educational interventions were implemented for 4 months and the questionnaire was subsequently repeated. The first educational intervention was a poster reminding junior trainees to draw a box heart and to review the echocardiogram for the newly admitted patients they clerked. The second educational intervention was a junior-senior discussion on the cardiorespiratory pathophysiology and key findings on echocardiogram for the newly admitted patients they clerked. The results were analysed using percentages.

Results Following the implementation of the two interventions, the proportion of junior trainees who drew a box heart and reviewed the echocardiogram of patients they clerked increased. Junior trainees reported a higher level of confidence in their understanding of the cardiorespiratory physiology of patients they clerked, and in identifying their key findings on echocardiogram. The reported combined time taken to draw a box heart and review the echocardiogram did not exceed fifteen minutes.

Conclusions Drawing a box heart and reviewing the echocardiogram with senior support are two quick methods to increase the educational aspects of clerking ward admissions for junior trainees on a paediatric cardiology ward.

\section{THE KEY BARRIERS TO THE UPTAKE OF PMTCT SERVICES IN UGANDA}

Emily Operto. UK

\subsection{6/bmipo-2021-RCPCH.32}

Background Mother-to-child transmission of the human immunodeficiency virus (HIV) is the second leading cause of new HIV infections among children in Uganda. However, approximately $40-50 \%$ of eligible women in Uganda do not use 3 of the key services provided by a programme of interventions to prevent this route of HIV transmission (PMTCT). A report on the global success of the prevention of mother-to-child transmission programme found that barriers exist at individual community levels to accessing these services.

Objectives The aim of the review is to explore the key barriers to the uptake of services in component 3 of the prevention of mother-to-child transmission programme by women in Uganda.

Methods A systematic, focussed literature search was carried out using relevant databases. Inclusion criteria were used to select relevant studies.

Results The review identified a number of key barriers that affected uptake of services provided by component 3. Gender norms limit the ability of HIV-positive women to make decisions about accessing and accepting HIV testing and anti-retroviral prophylaxis. HIV-related stigma poses a significant deterrent from accessing both of these services, and adhering to anti-retroviral regimens. Low quality of HIV and infant feeding counselling may affect acceptance and adherence to HIV testing and anti-retroviral prophylaxis. A limited amount of literature was available discussing factors that influenced infant feeding practices among HIV-positive mothers however studies which had been carried out suggested that cultural norms are the strongest barrier to the acceptance of exclusive breastfeeding or exclusive replacement feeding for the first 6 months of the infant's life.

Conclusions Women eligible for the prevention of mother-tochild transmission programme are in need of further support to improve their access and adherence to services. A number of strategies have been suggested to address these barriers.

\section{CLINICAL AUDIT ON THE MANAGEMENT OF KAWASAKI DISEASE}

Emily Operto, Filip Kucera. UK

\subsection{6/bmjpo-2021-RCPCH.33}

Background Kawasaki disease is a systemic vasculitis of unknown etiology that can result in coronary artery aneurysms in up to $25 \%$ of patients. ${ }^{2}$ This an important cause of morbidity and mortality in this condition. ${ }^{1}$ In the longer term, patients with persistent coronary artery aneurysms can develop 